\title{
ERRATA
}

\section{Erratum: Separation of germanium and silicon oxides by plasma-chemical deposition of germanosilicate glass in a moving plasma column [Tech. Phys. Lett. 25, 530-532 (1999)]}

K. M. Golant and I. V. Nikitin

Scientific Center of Fiber Optics, Institute of General Physics, Academy of Sciences, Moscow

[S1063-7850(99)03212-7]

The correct author's name is I. V. Nikolin. We apologize for this error and are reprinting the article in its entirety.

\section{Separation of germanium and silicon oxides by plasma-chemical deposition of germanosilicate glass in a moving plasma column}

\author{
K. M. Golant and I. V. Nikolin \\ Scientific Center of Fiber Optics, Institute of General Physics, Russian Academy of Sciences, Moscow \\ (Submitted April 5, 1999) \\ Pis'ma Zh. Tekh. Fiz. 25, 55-61 (July 12, 1999) \\ The optical transmission spectrum of germanosilicate glass deposited by surface plasma chemical \\ vapor deposition on the inner surface of a quartz tube revealed interference resonances \\ typical of multilayer dielectric coatings with alternating refractive indices. It is shown that this \\ effect can be attributed to the longitudinal inhomogeneity of the plasma composition and \\ specifically to an axial shift of the concentration maxima of germanium and silicon oxide. As a \\ plasma having a nonuniform composition moves along the tube, a layer of glass is formed \\ with a strong transverse germanium concentration gradient. It is established that in surface plasma \\ chemical vapor deposition the axial separation of the regions of deposition of the silicon \\ and germanium oxides increases if the glass is synthesized under conditions of oxygen deficiency. \\ (C) 1999 American Institute of Physics. [S1063-7850(99)01107-6]
}

Surface plasma chemical vapor deposition (SPCVD) is used to synthesize quartz-glass preforms for fiber-optical waveguides. ${ }^{1}$ In this method, layers of pure and doped $\mathrm{SiO}_{2}$ are deposited on the inner surface of a section of quartz glass tube whose volume is periodically filled with a chemically active plasma. This periodic filling is accomplished by varying the length of a steady-state plasma column which is created and sustained in the tube by microwave energy transported by surface plasma waves. ${ }^{2}$ The length of the column (distance between the point of application of the microwave field and the point of plasma detachment) is regulated by varying the power supplied to the plasma.

In SPCVD, silicon dioxide is synthesized by passing silicon tetrachloride mixed with oxygen through a moving plasma column. When the reagents enter the discharge zone, gas-phase $\mathrm{SiO}$ molecules are formed as a result of the plasma-chemical interaction between $\mathrm{SiCl}_{4}$ and oxygen molecules excited by electron impact. The process of glass for- mation on the walls of the tube terminates, where the $\mathrm{SiO}$ adsorbed from the gas phase is oxidized to give $\mathrm{SiO}_{2}$ by heterogeneous addition of oxygen. The chlorine released as a result of the reactions and the excess oxygen are removed by the pumping system.

Here we report some characteristic features of the formation of germanosilicate glass by this method. In the experiments we synthesized and investigated glass coatings $200 \mu \mathrm{m}$ thick deposited on the inner surface of a quartz tube having an outer diameter of $20 \mathrm{~mm}$ and a wall thickness of $2 \mathrm{~mm}$. An $\mathrm{O}_{2}+\mathrm{SiCl}_{4}+\mathrm{GeCl}_{4}$ mixture was passed into the tube at a total pressure of around 0.2 Torr. Each reagent was supplied via a separate RRG-9 gas flow rate regulator which measured each component of the gas mixture to within $1 \%$. The coatings were deposited by periodically moving a plasma along a $25 \mathrm{~cm}$ long section of the tube. The velocity and amplitude of the displacement of the plasma column front was controlled using a feedback system which controls 


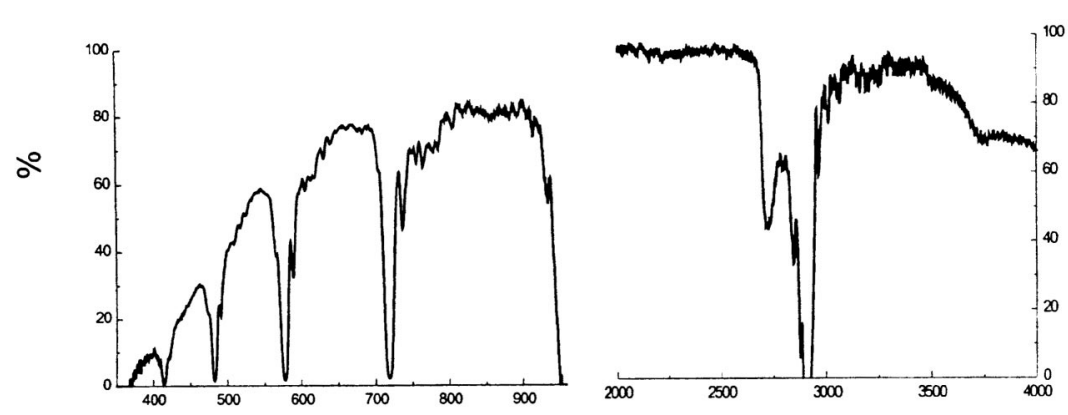

FIG. 1. Transmission spectrum of a germanosilicate glass coating $200 \mu \mathrm{m}$ thick deposited on the inner surface of a quartz glass tube by SPCVD. The measurements were made using an S2000 Ocean Optics Inc. spectrometer at 350-940 $\mathrm{nm}$ and using a Perkin-Elmer Fourier spectrometer at $2000-4000 \mathrm{~nm}$.

$\lambda, n m$

the microwave power supplied to the plasma. The chloride flow rate and the plasma displacement velocity were such that a $200 \mu \mathrm{m}$ thick coating was obtained after 400 plasma passes. The temperature of the tube walls was stabilized at around $1200{ }^{\circ} \mathrm{C}$ by means of an electrical furnace and was monitored with an optical pyrometer. During the deposition process we observed temperature fluctuations in the range of $\pm 50{ }^{\circ} \mathrm{C}$ about a given value, which were synchronous with the periodic motion of the plasma column and caused by the internal plasma heating of the tube. The deposition process was carried out with a flow rate ratio $\mathrm{SiCl}_{4} / \mathrm{GeCl}_{4}=10: 1$, which corresponds to a $\mathrm{GeO}_{2}$ content in the resultant glass of around $10 \mathrm{~mol} \%$ assuming $100 \%$ efficiency of implanting silicon and germanium atoms in the glass. After the end of the deposition process, the tube together with the layer of germanosilicate glass deposited on the inside was cut into transverse sections. These transverse sections were used as samples to obtain transmission spectra and were examined by electron microscopy.

A characteristic feature of the optical transmission spectrum of the deposited glass is its contrast interference structure (Fig. 1) which indicates that the refractive index distribution exhibits regular variations over the coating thickness. From the spectral position of the peaks we can readily establish that these correspond to different interference orders of the same periodic refractive-index structure with the fundamental interference order at the wavelength $\lambda_{0} \approx 2.9 \mu \mathrm{m}$.

Figure 2 shows a photograph of a transverse section of the deposited layer of germanosilicate glass obtained using a scanning electron microscope. The light bands correspond to sections of the glass having an increased germanium content. It can be seen that the periodic structure responsible for the observed transmission spectrum of the coating can be attributed to germanium concentration peaks repeated from one layer to another, which increase the refractive index of the glass. Note that, judging by Fig. 2, the thickness of the germanium-enriched layers is small and is at least an order of magnitude less than the period of the structure $(\approx 1 \mu \mathrm{m}$ for the deposition regime described above). We used the method of characteristic matrices ${ }^{3}$ to model the transmission spectrum of a structure formed by "thick" dielectric layers of thickness $d$ separated by "thin" dielectric layers of high re- fractive index. The fundamental interference order for which the transmission coefficient of this structure has a minimum corresponds to the condition $d=\lambda_{0} / 2 n$ where $n$ is the refractive index of the glass in the thick layer. For $\lambda_{0} \approx 2.9 \mu \mathrm{m}$ and $n \approx 1.45$ (undoped quartz glass) we obtain the estimate $d$ $\approx 0.98 \mu \mathrm{m}$ which shows good agreement with the period of the layered structure shown in Fig. 2. If the total number of structure periods is 200 and the thickness of the thin germanium-enriched layers is of the order of $0.1 \mu \mathrm{m}$, the refractive index of the thin layers would have to be 1.55-1.6 to produce a contrast interference spectrum such as that shown in Fig. 1. Since the refractive index increment of quartz glass caused by introducing $10 \mathrm{~mol} \% \mathrm{GeO}_{2}$ does not exceed 0.014, it must be concluded that the $\mathrm{SiO}_{2}$ and $\mathrm{GeO}_{2}$ are substantially stratified at the glass formation stage in SPCVD. We note that the observed effect is too great to be a consequence of variations in the germanium content caused by the periodic temperature fluctuations of the tube walls noted above.

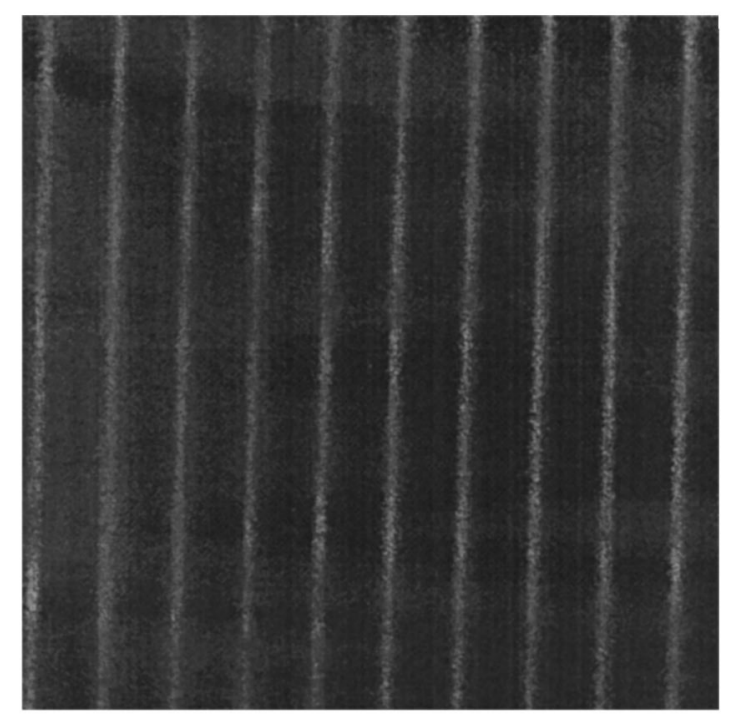

FIG. 2. Electron image of the transverse cross section of a deposited coating, which illustrates the periodic spatial variation of the germanium distribution. The period of the structure is $1 \mu \mathrm{m}$. 


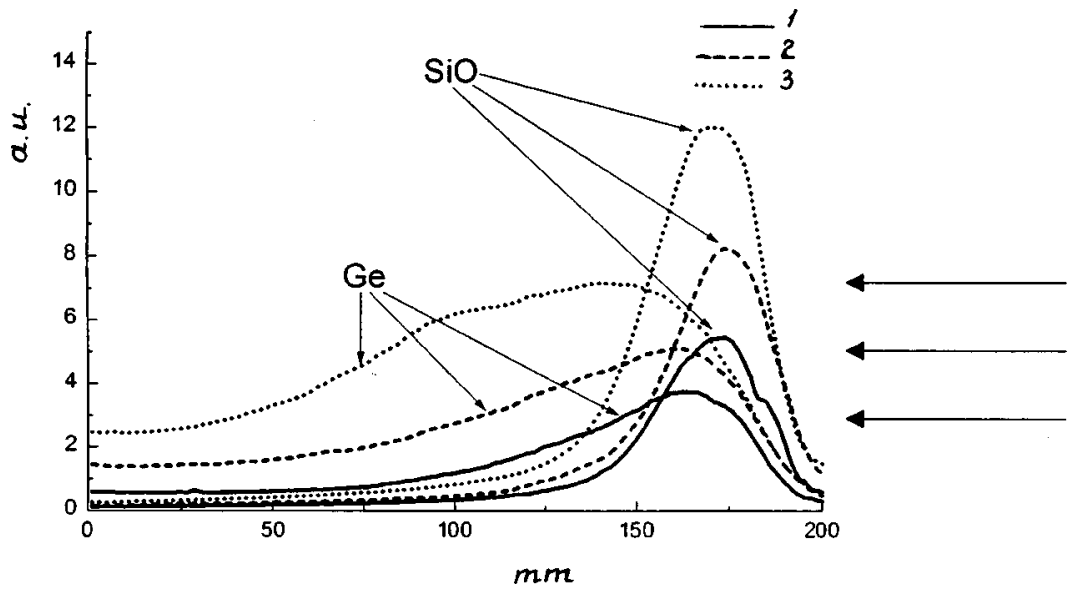

FIG. 3. Intensity distribution of the emission lines of $\mathrm{SiO}$ molecules and $\mathrm{Ge}$ atoms along a chemically active plasma at various oxygen partial pressures. The excess of oxygen in the gas mixture over that required by stoichiometry to oxidize all the silicon and germanium atoms is: $1-$ a factor of $6.5 ; 2-$ a factor of 4 ; and $3-$ a factor of 2.6. The abscissa is directed from the exciter toward the point of plasma quenching.
Direct confirmation of a layering effect at the stage where the chlorides are converted to the gas phase is provided by the distribution curves of $\mathrm{SiO}$ molecules and $\mathrm{Ge}$ atoms in the plasma, obtained by local emission optical spectroscopy (Fig. 3). In the experiments we measured the intensity distributions of the $425 \mathrm{~nm}$ ( $\mathrm{SiO}$ molecular band ${ }^{4}$ ) and the $327 \mathrm{~nm}$ spectral lines (Ge atomic line ${ }^{5}$ ) along the plasma column. Apart from variations in the parameters of the plasma electron component (density and temperature), the radiation intensities of the bands correspond to the concentrations of emitting particles. It can be seen from Fig. 3 that the maxima of the axial distributions of $\mathrm{SiO}$ and $\mathrm{Ge}$ are shifted relative to each other and the shift increases as the oxygen partial pressure decreases. As a result of this shift, the gaseous medium above the deposition zone is a section of plasma having a highly nonuniform composition. On its right-hand side some distance from the microwave exciter, predominantly $\mathrm{SiO}_{2}$ is deposited whereas on the left-hand side it is predominantly $\mathrm{GeO}_{2}$ (Fig. 2). The periodic motion of this inhomogeneous deposition zone relative to the tube results in the formation of a layered structure in the deposited layer.

Note that the thermodynamic characteristics of $\mathrm{SiO}$ and $\mathrm{GeO}$ differ substantially. For instance, the sublimation temperature of $\mathrm{GeO}$ at normal pressure is only $710^{\circ} \mathrm{C}$ (Ref. 6), i.e., even at high pressure it is far below the characteristic temperature maintained during the deposition of quartz glass in the SPCVD process $\left(1000-1200^{\circ} \mathrm{C}\right)$. This can explain the "pulling" of Ge from the deposition zone into the depth of the plasma column. Quite clearly, this effect is intensified when there is an oxygen deficiency because the rate of heterogeneous oxidation of $\mathrm{GeO}$ to form $\mathrm{GeO}_{2}$ is reduced.
To conclude we note that this layering effect in SPCVD is evidently typical not only of germanosilicate glass but also of other $\mathrm{SiO}_{2}$-based systems in which the active radicals forming the glass are produced in different parts of the deposition zone. By varying the ratio of the reagent flow rates, we can control the parameters of these layered structures so that this effect can be used to produce quartz-glass optical interference filters.

For the optical measurements we used apparatus from the central pool of spectroscopic equipment of the Russian Academy of Sciences and we should like to thank the director, V. G. Plotnichenko, for making this possible. The authors would also like to thank S. V. Lavrishchev for the electron microscopic analyses of the samples and Prof. A. S. Biryukov for valuable comments.

This work was partially supported by the Russian Fund for Fundamental Research (Project No. 98-02-16361).

${ }^{1}$ D. Pavy, M. Moisan, S. Saada, P. Chollet, P. Leprince, and J. Marrec, in Proceedings of the 12th European Conference on Optical Communication, Barcelona, 1986, pp. 19-22.

${ }^{2}$ M. Moisan, C. Beaudry, and P. Leprince, IEEE Trans. Plasma Sci. PS3(2), 55 (1975).

${ }^{3}$ M. Born and E. Wolf, Principles of Optics, 4th ed. (Pergamon Press, Oxford, 1969; Nauka, Moscow, 1970, 885 pp.).

${ }^{4}$ K. P. Huber and G. Herzberg, Molecular Spectra and Molecular Structure, Vol. 4 Constants of Diatomic Molecules [Russ. transl., Nauka, Moscow, 1984, 365 pp.].

${ }^{5}$ C. H. Corliss and W. R. Bozeman, Experimental Transition Probabilities for Spectral Lines of Seventy Elements (U.S. Government Printing Office, Washington, D.C., 1962; Mir, Moscow, 1968, 561 pp.).

${ }^{6}$ Handbook of Physical Quantities edited by I. S. Grigor'ev and E. Z. Merlikhov [in Russian], Energoatomizdat, Moscow (1991), 1232 pp.

Translated by R. M. Durham 